\title{
Use of N-Acetylcysteine in Amphetamine-Induced Acute Liver Failure
}

\author{
Saif Affas ${ }^{\mathrm{a}, \mathrm{c}}$, Mohamad Fekredeen Ayas ${ }^{\mathrm{a}, \mathrm{c}, \mathrm{d}}$, Ihab A. Kassab ${ }^{\mathrm{b}}$
}

\begin{abstract}
Acute liver failure (ALF) is a serious complication of many drugs. Amongst recreational drugs, cocaine, amphetamines and ecstasy (methylenedioxymethamphetamine) have been known to cause ALF as a complication. However, the true effects and management on the liver of such cases have not been well reported and treatment of such conditions needs prompt action. N-acetylcysteine (NAC) is a known hepatoprotective agent but remains controversial in the use of recreational drug-induced acute liver injury. We present a case of ALF secondary to amphetamine ingestion, with a rapid recovery after administration of intravenous NAC.
\end{abstract}

Keywords: Acute liver failure; NAC; Amphetamine

\section{Introduction}

Amphetamine is a popular recreational drug in the western world, as it induces euphoria and boosts self-confidence. Excessive doses can cause a wide variety of symptoms including, but not limited to increased state of arousal, euphoria, increased energy, talkativeness, agitation, visual hallucinations, cardiovascular manifestations and hyperthermia [1, 2]. Moreover, hepatotoxicity has been reported in the literature with no clear treatment recommendations [3].

$\mathrm{N}$-acetylcysteine (NAC) is a known medication used in the treatment of acetaminophen-induced hepatitis as well as other conditions, such as acute respiratory distress syndrome, and the use in influenza as well as other viral diseases such as COVID-19-induced hepatitis, and its use in cocaine-related liver injury; however, all still remains controversial [4-7]. NAC

Manuscript submitted October 23, 2020, accepted November 14, 2020

Published online December 30, 2020

aDepartment of Internal Medicine, Ascension St. John Hospital, Detroit, MI, USA

bivision of Hospital Medicine, Department of Internal Medicine, University of Michigan, Ann Arbor, MI, USA

'These authors contributed equally to this work.

${ }^{\mathrm{d} C}$ Corresponding Author: Mohamad Fekredeen Ayas, Department of Internal Medicine, Ascension St. John Hospital, 22101 Moross Rd, Detroit, MI 48236, USA. Email: Mohamad.ayas@ascension.org

doi: https://doi.org/10.14740/jmc3611 works as a hepatoprotective agent via multiple mechanisms, including restoring hepatic glutathione (GSH), and serving as a GSH substitute [8, 9].

\section{Case Report}

A 20-year-old man with no significant past medical history presented with palpitations after ingesting $2 \mathrm{~g}$ of methamphetamine. He soon after became obtunded and was intubated for airway protection. He was later found to be hypertensive with a pressure of 160/86 $\mathrm{mm} \mathrm{Hg}$, hyperthermic with a temperature of $42{ }^{\circ} \mathrm{C}$ and tachycardic with a heart rate of 112 beats per minute. A few hours later, the patient was found to be hypotensive with a pressure of $82 / 59 \mathrm{~mm} \mathrm{Hg}$ with minimal response to fluids, central line was placed and patient was started on norepinephrine. Physical exam showed an obtunded individual, with normal breath sounds in bilateral lung fields and an increased heart rate, with normal rhythm without murmurs.

On admission, the patient's urine toxicology was positive for amphetamines, and laboratory findings (labs) showed an aspartate transaminase (AST) of $121 \mathrm{IU} / \mathrm{L}$ with an alanine transaminase (ALT) of $32 \mathrm{U} / \mathrm{L}$ and a protime/prothrombin time (PT) of $19.4 \mathrm{~s}$ with a partial thromboplastin time (PTT) of 36.5 $\mathrm{s}$ with an international normalized ratio (INR) of 1.61, and a normal bilirubin.

Poison control was contacted and recommended supportive care with the initiation of NAC. Intravenous (IV) NAC loading dose followed by a maintenance dose was started around $6 \mathrm{~h}$ after admission.

Within $24 \mathrm{~h}$ of admission, the patient started to develop multi-organ failure including ALF with an AST of 1,324 IU/L, an ALT of $217 \mathrm{U} / \mathrm{L}$, a PT of $25.8 \mathrm{~s}$, a PTT of $43 \mathrm{~s}$, an INR of 2.32 and a bilirubin of $0.6 \mathrm{mg} / \mathrm{dL}$. On day 3 of hospital admission, the patient's labs showed a bilirubin of $1.6 \mathrm{mg} / \mathrm{dL}$, an AST of 2,350 IU/L, an ALT of 2,145 U/L, an INR of 3.19, a PTT of $44.8 \mathrm{~s}$ and a PT of $33.1 \mathrm{~s}$. Conversely, his serum total protein and serum albumin reached a nadir around the same time period.

After 3 - 4 days of NAC therapy, there was a significant recovery in liver enzymes. The starting of normalization of AST, ALT and PT/INR/PTT was observed by day 5 .

\section{Discussion}

Hepatotoxicity secondary to recreational drug use, such as cocaine, methylenedioxymethamphetamine (MDMA) and am- 
phetamines, has been documented in the literature, but no clear recommendations on its management have yet been given [3, $6]$. Although dialysis is a very effective method of removing the drugs, NAC has shown promising effects in the management of ALF secondary to drug use [6]. Studies have shown that NAC has a hepatoprotective effect and is suggested to be via multiple mechanisms of action, which includes antioxidant and anti-inflammatory effects [10-13]. The antioxidant ability of NAC to reduce the effect of oxidative stress is thought to be due to NAC's ability to decrease free radicals via replenishment of depleted intra-hepatocytes GSH, and the stimulation of a few enzymes, including superoxide dismutase (SOD), glutathione peroxidase (GPx) and catalase (CAT) [11]. It had been previously reported in a study conducted on the hepatocytes of rats, that NAC has been used to protect from cocaineinduced liver injury by up-regulating antioxidant enzymes, such as SOD [14]. NAC also has an anti-inflammatory effect, by decreasing the level of inflammatory cytokines released, and blocking tumor necrosis factor (TNF) alpha activation by modulating TNF alpha receptors [13]. On a cellular level, Lcysteine (L-cys) is a tripeptide ( $\gamma$-glutamyl cysteinyl glycine) and is the rate-limiting amino acid needed for GSH synthesis, but it is poorly penetrated into the cells. NAC however, easily penetrates the cells and is then deacetylated into L-cys, thereby promoting GSH synthesis for reducing the effect of the oxidative stress that is responsible for the liver injury [9].

\section{Conclusions}

Although not yet widely tested on human subjects, NAC has been shown to have anti-inflammatory effects that may help with drug-induced liver injury. It was successfully used in treating our patient's amphetamine-induced acute hepatic failure. Therefore, more studies need to be conducted to investigate the extended role of NAC in treating such cases of acute liver injury/failure.

\section{Acknowledgments}

None to declare.

\section{Financial Disclosure}

None to declare.

\section{Conflict of Interest}

None to declare.

\section{Informed Consent}

Informed consent was granted by the patient prior to writing this manuscript.

\section{Author Contributions}

Saif Affas: literature review, preparation, writing and editing of entire manuscript. Mohamad Fekredeen Ayas: literature review, preparation, writing and editing of entire manuscript. Ihab A. Kassab: literature review, preparation and writing part of discussion section.

\section{Data Availability}

Information was collected from a PubMed search using the keywords "Amphetamines, $\mathrm{N}$-acetylcysteine, acute liver failure". All other data were collected from the eCare system of Ascension St. John Hospital.

\section{References}

1. Steinkellner T, Freissmuth M, Sitte HH, Montgomery T. The ugly side of amphetamines: short- and long-term toxicity of 3,4-methylenedioxymethamphetamine (MDMA, 'Ecstasy'), methamphetamine and D-amphetamine. Biol Chem. 2011;392(1-2):103-115.

2. Vasan S, Olango GJ. Amphetamine toxicity. In: StatPearls [Internet]. Treasure Island (FL): StatPearls Publishing; 2020.

3. Jones AL, Simpson KJ. Review article: mechanisms and management of hepatotoxicity in ecstasy (MDMA) and amphetamine intoxications. Aliment Pharmacol Ther. 1999;13(2):129-133.

4. De Flora S, Balansky R, La Maestra S. Rationale for the use of $\mathrm{N}$-acetylcysteine in both prevention and adjuvant therapy of COVID-19. FASEB J. 2020;34(10):1318513193.

5. Pacht ER, Timerman AP, Lykens MG, Merola AJ. Deficiency of alveolar fluid glutathione in patients with sepsis and the adult respiratory distress syndrome. Chest. 1991;100(5):1397-1403.

6. Ansari M, Arshed S, Islam M, Sen S, Yousif A. A case of reversible drug-induced liver failure. Clin Case Rep. 2017;5(7):1181-1183.

7. De Flora S, Grassi C, Carati L. Attenuation of influenzalike symptomatology and improvement of cell-mediated immunity with long-term N-acetylcysteine treatment. Eur Respir J. 1997;10(7):1535-1541.

8. de Andrade KQ, Moura FA, dos Santos JM, de Araujo OR, de Farias Santos JC, Goulart MO. Oxidative stress and inflammation in hepatic diseases: therapeutic possibilities of N-acetylcysteine. Int J Mol Sci. 2015;16(12):3026930308 .

9. Lu SC. Regulation of glutathione synthesis. Mol Aspects Med. 2009;30(1-2):42-59.

10. Arranz L, Fernandez C, Rodriguez A, Ribera JM, De la Fuente M. The glutathione precursor N-acetylcysteine improves immune function in postmenopausal women. Free Radic Biol Med. 2008;45(9):1252-1262.

11. Caglikulekci M, Pata C, Apa DD, Dirlik M, Tamer L, 
Yaylak F, Kanik A, et al. The effect of N-acetylcysteine (NAC) on liver and renal tissue inducible nitric oxide synthase (iNOS) and tissue lipid peroxidation in obstructive jaundice stimulated by lipopolysaccharide (LPS). Pharmacol Res. 2004;49(3):227-238.

12. Hayakawa M, Miyashita H, Sakamoto I, Kitagawa M, Tanaka H, Yasuda H, Karin M. Evidence that reactive oxygen species do not mediate NF- $\mathrm{KB}$ activation. EMBO J. 2003;22:3356-3366.
13. Hou Y, Wang L, Yi D, Ding B, Yang Z, Li J, Chen X, et al. N-acetylcysteine reduces inflammation in the small intestine by regulating redox, EGF and TLR4 signaling. Amino Acids. 2013;45(3):513-522.

14. Zaragoza A, Diez-Fernandez C, Alvarez AM, Andres D, Cascales M. Effect of N-acetylcysteine and deferoxamine on endogenous antioxidant defense system gene expression in a rat hepatocyte model of cocaine cytotoxicity. Biochim Biophys Acta. 2000;1496(2-3):183-195. 